\title{
System For Finding Location Domination Number Of A Graph By The Fusion Of Vertex
}

\author{
Rajasekar. $\mathrm{G}^{1}$, Nagarajan. $\mathrm{K}^{2}$ \\ Department of Mathematics ${ }^{1,2}$, Jawahar Science College, Neyveli ${ }^{1,2}$ \\ Email:grsmaths@gmail.com ${ }^{1}$,n2a4g1r1j1@gmail.com ${ }^{2}$
}

\begin{abstract}
Locating-Dominating set ( $L D$-set) of a graph $G$, is a dominating set $S$ with the property that every vertices $v$ in $V(G)-S$ can be identified uniquely by the set of neighbours of $v$ which are in $S$. In this paper a procedure for finding the locating-domination number of graph obtained by fusion of a single vertex from two graphs has been formulated. By this procedure the location domination number of $C_{m} \bowtie_{f} C_{n}$, Windmill graph, Dutch windmill graph and Friendship graph are been found.
\end{abstract}

Keywords-Dominating set; Locating set; Locating domination set; Fusion of vertex

\section{INTRODUCTION}

For any vertex $v$ in $V(G)-S, S(v)$ is the set of vertices in $S$ which are adjacent to $v$. Locatingdominating set was introduced by Slater $[1,2]$ which is defined as follows. A dominating set $S$ is defined to be a locating-dominating set if $S(v) \neq S(w)$, for any $v, w \in V(G)-S$. A locating dominating set is denoted by $L D$-set. The minimum cardinalities of an $L D$-set in $G$ is called the location-domination number of $G$ and it is denoted by $R D(G)$. An $L D$ set with $R D(G)$ elements is called as a referencingdominating set or an $R D$-set. The set of all $R D$-set of the given graph is denoted by $\mathscr{S}$.

G. Rajasekar et al $[3,4,5]$ have found location domination number of sum of graphs, graph connected by bridge and graph obtained by fusion of vertex.

The windmill graph $K_{n}^{(m)}$ or $W d(n, m)$ is the graph obtained by taking $m$ copies of the complete graph $K_{n}$ with a vertex in common. The Dutch windmill graph $D_{n}^{(m)}$ is the graph obtained by taking $m$ copies of the cycle $C_{n}$ with a vertex in common.

\section{PRELIMINARIES}

Let $G$ be the graph obtained from $G_{1}$ and $G_{2}$ by vertex fusion of vertices $v_{1} \in G_{1}$ and $v_{2} \in G_{2}$ to form a single new vertex $v$. Let us denote this graph $G$ by $G=G_{1}\left\{v_{1}\right\} \bowtie_{f} G_{2}\left\{v_{2}\right\}$.

Theorem 2.1. [4]: Let $G_{1}, G_{2}$ be any two graphs and $G$ be a graph obtained by fusion of vertices $v_{1} \in G_{1}$ and $v_{2} \in G_{2}$ to form a single vertex $v$, then $R D\left(G_{1}\right)+R D\left(G_{2}\right)-2 \leq R D(G) \leq R D\left(G_{1}\right)+R D\left(G_{2}\right)$

Theorem 2.2. [4]: Let $G_{1}, G_{2}$ be any two graphs and $G=G_{1}\left\{v_{1}\right\} \bowtie_{\mathrm{f}} G_{2}\left\{v_{2}\right\}$. For $i=1,2$, let $G_{i}$ have an $R D$-set $S_{i}$ such that $v_{i} \in S_{i}$ and $S_{i}\left(u_{i}\right) \neq\left\{v_{i}\right\}$ for all $u_{i} \in V\left(G_{i}\right)-S_{i}$.then $R D(G)=R D\left(G_{1}\right)+R D\left(G_{2}\right)-2$

if and only if for $i=1,2, S_{i}-\left\{v_{i}\right\}$ is the $R D$-set of $G_{i}-\left\{v_{i}\right\}$ and $N_{G_{i}}\left(v_{i}\right) \cap S_{i} \neq \Phi$.

Theorem 2.3. [4]: Let $G_{1}, G_{2}$ be any two graphs and $G=G_{1}\left\{v_{1}\right\} \bowtie_{\mathrm{f}} G_{2}\left\{v_{2}\right\}$. If for both $i=1$ and $2 v_{i}$ does not belongs to anyone of the $R D$-set of $G_{i}$ then $R D(G)=R D\left(G_{1}\right)+R D\left(G_{2}\right)$.

Theorem 2.4. [4]: Let $G_{1}, G_{2}$ be any two graphs and $G=G_{1}\left\{v_{1}\right\} \bowtie_{\mathrm{f}} G_{2}\left\{v_{2}\right\}$. Let either $G_{1}$ or $G_{2}$ has atleast one $R D$-set $S_{1}$ or $S_{2}$ respectively such that $v_{1} \in S_{1}$ or $v_{2} \in S_{2}$ (say $v_{1} \in S_{1}$ ). If $S_{1}-\left\{v_{1}\right\}$ is the $R D$-set of $G_{1}-\left\{v_{1}\right\}$ for some $R D$-set $S_{1}$ then $R D(G)$ will be equal $R D\left(G_{1}\right)+R D\left(G_{2}\right)-1$ otherwise $R D(G)$ will be equal $R D\left(G_{1}\right)+R D\left(G_{2}\right)$.

Theorem 2.5. [4]: Let $G_{1}, G_{2}$ be any two graphs and $G=G_{1}\left\{v_{1}\right\} \bowtie_{\mathrm{f}} G_{2}\left\{v_{2}\right\}$. For $i=1,2$, let $G_{i}$ have an $R D$-set $S_{i}$ such that $v_{i} \in S_{i}$. If for every $R D$-set $S_{i}$, there exist a vertex $u_{i} \in V\left(G_{i}\right)-S_{i}$ such that $S_{i}\left(u_{i}\right)=\left\{v_{i}\right\} \quad$ for both $i=1$ and 2 then $R D(G)=R D\left(G_{1}\right)+R D\left(G_{2}\right)$.

Theorem 2.6. [4]: Let $G_{1}, G_{2}$ be any two graphs and $G=G_{1}\left\{v_{1}\right\} \bowtie_{f} G_{2}\left\{v_{2}\right\}$. For $i=1,2$, let $G_{i}$ have an $R D$-set $S_{i}$ such that $v_{i} \in S_{i}$. If $G_{2}$ has an $R D$-set $S_{2}$ such that $S_{2}\left(u_{2}\right) \neq\left\{v_{2}\right\}$ for all $u_{2} \in V\left(G_{2}\right)-S_{2}$ and $G_{1}$ have no $R D$-set $S_{1}$ such that $S_{1}\left(u_{1}\right) \neq\left\{v_{1}\right\}$ for all $u_{1} \in V\left(G_{1}\right)-S_{1}$ then

$$
R D(G)=R D\left(G_{1}\right)+R D\left(G_{2}\right)-1 .
$$

Remark 2.1. [4]: Let $G_{1}, G_{2}$ be any two graphs and $G=G_{1}\left\{v_{1}\right\} \bowtie_{\mathrm{f}} G_{2}\left\{v_{2}\right\}$. For $i=1,2$, let $G_{i}$ have an 
$R D$-set $S_{i}$ such that $v_{i} \in S_{i}$ and $S_{i}\left(u_{i}\right) \neq\left\{v_{i}\right\}$ for all $u_{i} \in V\left(G_{i}\right)-S_{i}$. For either $i=1$ or 2 if $G_{i}$ does not have any $R D$-set $S_{i}$ satisfying the both conditions

(i) $S_{i}-\left\{v_{i}\right\}$ is the $R D$-set of $G_{i}-\left\{v_{i}\right\}$

(ii) $N_{G_{i}}\left(v_{i}\right) \cap S_{i} \neq \Phi$

then $R D(G)=R D\left(G_{1}\right)+R D\left(G_{2}\right)-1$.

\section{LOCATION DOMINATION NUMBER OF}

A GRAPH BY THE FUSION OF VERTEX

Algorithm for finding location domination number of graph obtained by fusion of vertex is formulated with the aid of Theorem 2.2, 2.3, 2.4, 2.5, 2.6 and Remark 2.1. We see that location domination number of graph obtained by fusion of vertex depends on three feature of $R D$-set of the graph. The three properties are defined as follows:

Property 1: $G$ has an $R D$-set $S \in \mathscr{S}$ such that $v \in S$ and $S-\{v\}$ is the $R D$-set of $G-\{v\}$ where $v$ is the vertex considered for fusion.

Property 2: $G$ has atleast one $R D$-set $S \in \mathscr{S}$ such that $S(u) \neq\{v\}$ for all $u \in V(G)-S$ and $v$ is the considered for fusion.
Property 3: $G$ has an $R D$-set $S \in \mathscr{S}$ such that $v \in S$ and $N_{G}(v) \cap S \neq \Phi$ where $v$ is the vertex considered for fusion.

Based on these three properties we formulate a procedure in form of Table 1. and Table 2. to find the location domination number of graphs obtained by fusion of vertex.

Remark 3.1. Let $G=G_{1}\left\{v_{1}\right\} \bowtie_{\mathrm{f}} G_{2}\left\{v_{2}\right\}$ where $v_{1}$ and $v_{2}$ are fused to form a single vertex $v$. If one of the following condition is true then $R D$-set $S$ of $G$ doesn't contains the vertex $v$.

(i) Both $G_{1}$ and $G_{2}$ doesn't have any $R D$-set which contains the vertex $v_{1}$ and $v_{2}$ respectively

(ii) Both $G_{1}$ and $G_{2}$ have any $R D$-set satisfying Property 1, Property 2 and Property 3 simultaneously

Remark 3.2. If graph $G$ doesn't satisfy Property 1 then there is no need to check Property 3.

Table 1. Procedure for finding the location domination number of graphs obtained by fusion of vertex- Table 1

\begin{tabular}{|c|c|c|}
\hline$R D(G)$ & $v_{2} \notin S_{2}$ for all $S_{2} \in \mathscr{S}_{2}$ & \multicolumn{1}{c|}{$v_{2} \in S_{2}$ for some $S_{2} \in \mathscr{S}_{2}$} \\
\hline \multirow{1}{*}{$v_{1} \notin S_{1}$ for all $S_{1} \in \mathscr{S}_{1}$} & $R D\left(G_{1}\right)+R D\left(G_{2}\right)$ & $\begin{array}{l}\text { If } G_{2} \text { satisfies Property } 1 \text { then } \\
R D(G)=R D\left(G_{1}\right)+R D\left(G_{2}\right)-1 \\
\text { otherwise } \\
R D(G)=R D\left(G_{1}\right)+R D\left(G_{2}\right)\end{array}$ \\
\hline$v_{1} \in S_{1}$ for some $S_{1} \in \mathscr{S}_{1}$ & $\begin{array}{l}\text { If } G_{1} \text { satisfies Property } 1 \text { then } \\
R D(G)=R D\left(G_{1}\right)+R D\left(G_{2}\right)-1 \\
\text { otherwise } \\
R D(G)=R D\left(G_{1}\right)+R D\left(G_{2}\right)\end{array}$ & $\begin{array}{l}\text { Check Property 2 for the graph } \\
G_{1} \text { and } G_{2} \text {. And refer Table 2 }\end{array}$ \\
\hline
\end{tabular}

Table 2. Procedure for finding the location domination number of graphs obtained by fusion of vertex- Table 2

\begin{tabular}{|c|l|c|}
\hline$R D(G)$ & \multicolumn{1}{|c|}{$G_{2}$ satisfies Property 2 } & $G_{2}$ doesn't satisfy Property 2 \\
\hline & $\begin{array}{l}\text { If both graphs } G_{1} \text { and } G_{2} \text { have } R D \text {-sets } \\
\text { which satisfies Property 1, Property 2 } \\
\text { and Property 3 simultaneously then } \\
G_{1} \text { satisfies } \\
\text { Property 2 } \\
\text { otherwise } \\
R D(G)=R D\left(G_{1}\right)+R D\left(G_{2}\right)-2\end{array}$ & $R D(G)=R D\left(G_{1}\right)+R D\left(G_{2}\right)-1$ \\
\hline $\begin{array}{l}G_{1} \text { doesn't satisfy } \\
\text { Property 2 }\end{array}$ & \multicolumn{1}{|c|}{$R D(G)=R D\left(G_{1}\right)+R D\left(G_{2}\right)-1$} & $R D(G)=R D\left(G_{1}\right)+R D\left(G_{2}\right)$ \\
\hline
\end{tabular}




\section{LOCATION DOMINATION NUMBER OF} $C_{m} \bowtie_{\mathrm{f}} C_{n}$, WINDMILL GRAPH, DUTCH WINDMILL GRAPH AND FRIENDSHIP GRAPH

This section deals with finding location domination number of $C_{m} \bowtie_{\mathrm{f}} C_{n}$, Windmill graph, Dutch windmill graph and Friendship graph which are obtained by vertex fusion. So let us first study the properties of $C_{n}$ and $K_{n}$ related to vertex fusion.

Properties of $\boldsymbol{C}_{n}$ : Let the vertex label of $C_{n}$ be $v_{1}, v_{2}, \ldots, v_{n-1}, v_{n}$. For cycle it is always possible to have a $R D$-set which contains the vertex considered for fusion.

- For $n=4,\left\{v_{1}, v_{2}\right\},\left\{v_{2}, v_{3}\right\},\left\{v_{3}, v_{4}\right\}$ and $\left\{v_{4}, v_{1}\right\}$ are the possible $R D$-set. And Property 1 and Property 2 are not satisfied for any of the possible $R D$-set.

- For $n \equiv 0 \bmod 5$, i.e. $n=5 k, k \geq 1$. $\left\{v_{2}, v_{4}, v_{7}, v_{9}, \ldots, v_{5 k-8}, v_{5 k-6}, v_{5 k-3}, v_{5 k-1}\right\}$ is the only possible $R D$-set and it doesn't satisfies Property 1, Property 2 as well as Property 3.

- For $n \equiv 1 \bmod 5$, i.e. $n=5 k+1, k \geq 1$. $\left\{v_{2}, v_{4}, v_{7}, v_{9}, \ldots, v_{5 k-3}, v_{5 k-1}, v_{5 k+1}\right\}$ is a $R D$-set such that $\left\{v_{2}, v_{4}, v_{7}, v_{9}, \ldots, v_{5 k-3}, v_{5 k-1}\right\}$ is the $R D$-set of $C_{n}-\left\{v_{5 k+1}\right\} \quad$ and $S\left(v_{i}\right) \neq\left\{v_{5 k+1}\right\}$ for all $v_{i} \in V\left(C_{n}\right)-\left\{v_{2}, v_{4}, v_{7}, v_{9}, \ldots, v_{5 k-3}, v_{5 k-1}, v_{5 k+1}\right\}$.

Hence Property 1 and Property 2 is satisfied with respect to vertex $v_{5 k+1}$. Whereas none of the vertices in the set $\left\{v_{2}, v_{4}, v_{7}, v_{9}, \ldots, v_{5 k-3}, v_{5 k-1}, v_{5 k+1}\right\}$ are adjacent, so Property 3 is not satisfied.

- For $n \equiv 2 \bmod 5$, i.e. $n=5 k+2, k \geq 1$. $\left\{v_{2}, v_{4}, v_{7}, v_{9}, \ldots, v_{5 k-3}, v_{5 k-1}, v_{5 k+2}\right\}$ is a $R D$-set such that $S\left(v_{i}\right) \neq\left\{v_{1}\right\}$ for all $v_{i} \in V\left(C_{n}\right)-\left\{v_{2}, v_{4}, v_{7}, v_{9}, \ldots, v_{5 k-3}, v_{5 k-1}, v_{5 k+2}\right\}$.

Hence Property 2 is satisfied with respect to vertex $v_{1}$. Whereas Property 1 and Property 3 is not fulfilled.

- For $n \equiv 3 \bmod 5$, i.e. $n=5 k+3, k \geq 0$. $\left\{v_{2}, v_{4}, v_{7}, v_{9}, \ldots, v_{5 k-3}, v_{5 k-1}, v_{5 k+2}, v_{5 k+3}\right\}$ is a $R D$-set such that $v_{5 k+3}$ satisfies Property 1, Property 2 and Property 3

- For $n \equiv 4 \bmod 5$, i.e. $n=5 k+4, k \geq 1$. $S=\left\{v_{2}, v_{4}, v_{7}, v_{9}, \ldots, v_{5 k-3}, v_{5 k-1}, v_{5 k+2}, v_{5 k+4}\right\} \quad$ is a $R D$-set such that $S\left(v_{i}\right) \neq\left\{v_{5 k+4}\right\}$ for all $v_{i} \in V\left(C_{n}\right)-S$. Hence Property 2 is satisfied with respect to vertex $v_{5 k+4}$. But Property 1 and Property 3 is not fulfilled.

Properties of $C_{n}$ are concatenated in the following Table 3

Properties of $\boldsymbol{K}_{n}$ : Let the vertex label of $K_{n}$ be $v_{1}, v_{2}, \ldots, v_{n-1}, v_{n}$. For complete graph it is always possible to have a $R D$-set which contains the vertex considered for fusion.

For $n=2$, Property 1 , Property 2 and Property 3 fails.

Clearly for $n \geq 3, S=\left\{v_{1}, v_{2}, \ldots, \mathrm{v}_{n-2}, v_{n-1}\right\}$ is an $R D$-set such that $S-\left\{v_{1}\right\}$ is the $R D$-set of $K_{n}-\left\{v_{1}\right\}$ $S\left(v_{n}\right) \neq\left\{v_{1}\right\}, S-\left\{v_{1}\right\}$ is the $R D$-set of $K_{n}-\left\{v_{1}\right\}$ and $N_{G}\left(v_{1}\right) \cup S=\left\{v_{2}, v_{3}, \ldots, v_{n-2}, v_{n-1}\right\} \neq \Phi$. Thus $K_{n}$ satisfy Property 1 , Property 2 and Property 3 .

Theorem 4.1. Let $G=C_{m} \bowtie_{f} C_{n}$ then

$$
\begin{aligned}
& R D(G)= \\
& \left\{\begin{array}{r}
R D\left(C_{m}\right)+R D\left(C_{n}\right), \quad \text { if } m, n \equiv 0 \bmod 5 \text { or } \\
m, n=4 \text { or } \\
m \equiv 0 \bmod 5 \text { and } n=4 \text { or } \\
m=4 \text { and } n \equiv 0 \bmod 5 \\
R D\left(C_{m}\right)+R D\left(C_{n}\right)-2, \text { if } m, n \equiv 3 \bmod 5 \\
R D\left(C_{m}\right)+R D\left(C_{n}\right)-1, \text { otherwise }
\end{array}\right.
\end{aligned}
$$

Proof. In cycle $C_{n}$, as vertex considered for fusion is in the $R D$-set, it is enough to check Table 2 alone.

As $n \equiv 3 \bmod 5$ satisfies Property 1, Property 2 and Property 3 while $n \equiv 0 \bmod 5$ and $n=4$ doesn't satisfies Property 1, Property 2 and Property 3 we have that

Table 3. Properties of $C_{n}$ related to vertex fusion

\begin{tabular}{|l|c|c|c|c|}
\hline \multicolumn{1}{|c|}{$C_{n}$} & $\begin{array}{c}\text { Vertex considered for } \\
\text { fusion is in the } R D- \\
\text { set }\end{array}$ & Property 1 & Property 2 & Property 3 \\
\hline $\begin{array}{l}n \equiv 0 \bmod 5 \text { and } \\
n=4\end{array}$ & Yes & Not satisfied & Not satisfied & Not satisfied \\
\hline$n \equiv 1 \bmod 5$ & Yes & Satisfied & Satisfied & Not satisfied \\
\hline $\begin{array}{l}n \equiv 2 \bmod 5 \\
n \equiv 4 \bmod 5 \operatorname{except} n=4\end{array}$ & Yes & Not satisfied & Satisfied & Not satisfied \\
\hline$n \equiv 3 \bmod 5$ & Yes & Satisfied & Satisfied & Satisfied \\
\hline
\end{tabular}


Available online at www.ijrat.org

$$
\begin{aligned}
& R D(G)= \\
& \left\{\begin{aligned}
& R D\left(C_{m}\right)+R D\left(C_{n}\right), \text { if } m, n \equiv 0 \bmod 5 \text { or } \\
& m, n=4 \text { or } m \equiv 0 \bmod 5 \text { and } n=4 \text { or } \\
& m=4 \text { and } n \equiv 0 \bmod 5 \\
& R D\left(C_{m}\right)+R D\left(C_{n}\right)-2, \text { if } m, n \equiv 3 \bmod 5
\end{aligned}\right.
\end{aligned}
$$

From Table 2, it is clear that for all other cases $R D(G)$ will be equal to $R D\left(C_{m}\right)+R D\left(C_{n}\right)-1$.

Corollary 4.1. Let $F_{n}$ be the friendship graph then $R D\left(F_{n}\right)=n$.

Proof. $F_{2} \cong C_{3} \bowtie_{f} C_{3}$, hence by Theorem $4.1 R D\left(C_{3}\right.$ $\left.\bowtie_{f} C_{3}\right)=R D\left(C_{3}\right)+R D\left(C_{3}\right)-2=2 \quad$ and by Remark 3.1 the fused vertex will not be in any $R D$ set.

$F_{n}$ is obtained by fusion any vertex of $C_{3}$ to the vertex with maximum degree in $F_{n-1}$ and by Table 1 we have

$$
\begin{aligned}
& R D\left(F_{n}\right)=R D\left(F_{n-1}\right)+R D\left(C_{3}\right)-1 \\
& R D\left(F_{n-1}\right)=R D\left(F_{n-2}\right)+R D\left(C_{3}\right)-1 \\
& \vdots \\
& R D\left(F_{4}\right)=R D\left(F_{3}\right)+R D\left(C_{3}\right)-1 \\
& R D\left(F_{3}\right)=R D\left(F_{2}\right)+R D\left(C_{3}\right)-1=2+2-1=3
\end{aligned}
$$

Thus $R D\left(F_{n}\right)=n-1+2-1=n$.

Theorem 4.2. For the Windmill graph $K_{n}^{(m)}$,

$$
R D\left(K_{n}^{(m)}\right)=\left\{\begin{array}{ll}
m, & \text { if } n=2 \\
m(n-2), & \text { if } n \geq 3
\end{array} .\right.
$$

Proof. $K_{2}^{(m)} \cong S_{m}$ and hence

$$
R D\left(K_{2}^{(m)}\right)=R D\left(S_{m}\right)=m .
$$

For $n \geq 3, K_{n}$ satisfy all Property 1, Property 2 and Property 3 , hence

$$
\begin{aligned}
R D\left(K_{n}^{(2)}\right) & =R D\left(K_{n}\right)+R D\left(K_{n}\right)-2 \\
& =2(n-2)
\end{aligned}
$$

and by Remark 3.1 the fused vertex will not be in any $R D$-set.

$K_{n}^{(m)}$ is obtained by fusion any vertex of $K_{n}$ to the vertex with maximum degree in $K_{n}^{(m-1)}$ and by Table 1 we have

$$
\begin{aligned}
& R D\left(K_{n}^{(m)}\right)=R D\left(K_{n}^{(m-1)}\right)+R D\left(K_{n}\right)-1 \\
& R D\left(K_{n}^{(m-1)}\right)=R D\left(K_{n}^{(m-2)}\right)+R D\left(K_{n}\right)-1 \\
& \vdots \\
& R D\left(K_{n}^{(4)}\right)=R D\left(K_{n}^{(3)}\right)+R D\left(K_{n}\right)-1 \\
& R D\left(K_{n}^{(3)}\right)=R D\left(K_{n}^{(2)}\right)+R D\left(K_{n}\right)-1=2(n-2)+n-1-1 \\
& =3(n-2)
\end{aligned}
$$

Hence $R D\left(K_{n}^{(m)}\right)=m(n-2)$.

Theorem 4.3. For the Dutch windmill graph $D_{n}^{(m)}$,

$$
R D\left(D_{n}^{(m)}\right)= \begin{cases}m\left\lceil\frac{2 n}{5}\right\rceil, & \text { if } n \equiv 0 \bmod 5 \text { or } \\ m\left\lceil\frac{2 n}{5}\right\rceil-m, & \text { if } n \equiv 3 \bmod 5 \\ m\left\lceil\frac{2 n}{5}\right\rceil-(m-1), & \text { otherwise }\end{cases}
$$

Proof. As $D_{n}^{(2)} \cong C_{n} \bowtie_{f} C_{n}$, by Theorem 4.1 we have that

$$
R D\left(D_{n}^{(2)}\right)=\left\{\begin{array}{l}
2\left\lceil\frac{2 n}{5}\right\rceil, \quad \text { if } n \equiv 0 \bmod 5 \text { or } \\
2\left\lceil\frac{2 n}{5}\right\rceil-2, \text { if } n \equiv 3 \bmod 5 \\
2\left\lceil\frac{2 n}{5}\right\rceil-1, \text { otherwise }
\end{array}\right.
$$

$D_{n}^{(m)}$ is obtained by fusion of any vertex of $C_{n}$ to the vertex of $D_{n}^{(m-1)}$ with maximum degree.

Case 1: For $n \equiv 0 \bmod 5$ and $n=4, D_{n}^{(2)}$ and $C_{n}$ do not satisfies Property 2. Moreover $D_{n}^{(m-1)}$ also do not satisfies Property 2 for $n \equiv 0 \bmod 5$ and $n=4$. Hence we have

$$
\begin{aligned}
& R D\left(D_{n}^{(3)}\right)=R D\left(D_{n}^{(2)}\right)+R D\left(C_{n}\right)=3\left\lceil\frac{2 n}{5}\right\rceil \\
& \vdots \\
& R D\left(D_{n}^{(m-1)}\right)=R D\left(D_{n}^{(m-2)}\right)+R D\left(C_{n}\right)=(m-1)\left\lceil\frac{2 n}{5}\right\rceil \\
& R D\left(D_{n}^{(m)}\right)=R D\left(D_{n}^{(m-1)}\right)+R D\left(C_{n}\right)=m\left\lceil\frac{2 n}{5}\right\rceil
\end{aligned}
$$

Case 2: For $n \equiv 3 \bmod 5$, any $R D$-set of $D_{n}^{(2)}$ does not contains the vertex considered for fusion. As $C_{n}$ satisfies Property 1 for $n \equiv 3 \bmod 5$ and by Table 1 we have 


$$
\begin{aligned}
R D\left(D_{n}^{(3)}\right) & =R D\left(D_{n}^{(2)}\right)+R D\left(C_{n}\right)-1 \\
= & 3\left\lceil\frac{2 n}{5}\right\rceil-3 \\
\vdots & R D\left(D_{n}^{(m-1)}\right)=R D\left(D_{n}^{(m-2)}\right)+R D\left(C_{n}\right)-1 \\
& =(m-1)\left\lceil\frac{2 n}{5}\right\rceil-(m-1) \\
R D\left(D_{n}^{(m)}\right) & =R D\left(D_{n}^{(m-1)}\right)+R D\left(C_{n}\right)-1=m\left\lceil\frac{2 n}{5}\right\rceil-m
\end{aligned}
$$

Case 3: For all other possibilities we can similarly prove that $R D\left(D_{n}^{(m)}\right)=m\left\lceil\frac{2 n}{5}\right\rceil-(m-1)$.

\section{ACKNOWLEDGMENTS}

This research is supported by UGC scheme RGNF. Award letter F1-17.1/2014-15/RGNF-2014-15-SCTAM-80373/(SAIII/Website).

\section{REFERENCES}

[1] Slater, P. J. (1988): Dominating and reference sets in a graph, J. Math. Phys. Sci., 22, pp. 445-455.

[2] Slater, P. J. (1987): Dominating and location in acyclic in graphs, Networks, 17, pp. 55-64.

[3] Rajasekar, G.; Nagarajan, K. (2018): Algorithm for finding Location Domination Number of a Graph connected by a Bridge, Int. J. of Pure and Applied Math., 118 (6), pp. 313-321.

[4] Rajasekar, G.; Nagarajan, K. (2017): Location Domination Number of Graph obtained by the Fusion of Single Vertex, Global J. of Pure and Applied Math., 13 (9), pp. 4425-4436.

[5] Rajasekar, G.; Nagarajan, K. (2018): Location Domination Number of Sum of Graphs, Int. J. of Engineering \& Technology, 7 (4.10), pp. 852-856. 